\title{
NUMERICAL TOOLS FOR THREE DIMENSIONAL SIMULATIONS OF THE ROTATING DETONATION ENGINE IN COMPLEX GEOMETRIES
}

\author{
Karol Swiderski, Michal Folusiak \\ Arkadiusz Kobiera, Borys Lukasik, Piotr Wolanski \\ Institute of Aviation, Propulsion Department \\ Krakowska Av. 110/114, 02-256 Warsaw, Poland \\ tel.: +48228460011 , ext. 361, fax: +48228464432 \\ e-mail: karol.swiderski@ilot.edu.pl
}

\begin{abstract}
This paper describes the development of a computational code REFLOPS USG (REactive FLOw solver for Propulsion Systems on UnStructured Grids) based on the Favre averaged Navier-Stokes equations with chemical reactions for semi-ideal multicomponent gas to predict the structure and dynamics of three-dimensional unsteady detonation as it occurs in the Rotating Detonation Engine (RDE). This work provides an overview of second order accurate in time and space finite volume method applied to conservation equations and its implementation on unstructured self-adaptive tetrahedral or hexahedral three-dimensional cell-centred meshes. The inviscid fluxes are given by the Riemann solver and stabilization is ensured by the proper limiters inherited from the TVD theory or gradient based limiters. The stiff equations of chemical kinetics are solved by use of implicit DVODE (Double precision Variablecoefficient Ordinary Differential Equation solver, with fixed-leading-coefficient implementation) routine or by explicit Chemeq 2 routine. Additional improvements are incorporated into the code such as parallelization in OpenMP and implementation of NVIDIA CUDA technology. REFLOPS USG has become a fundamental numerical tool in the research of $R D E$ at the Institute of Aviation in Warsaw, in frame of Innovative Economy project UDA-POIG.01.03.01-14-071 'Turbine engine with detonation combustion chamber' supported by EU and Ministry of Regional Development, Poland. The simulations presented in this paper are based on inviscid or viscous multicomponent semi-ideal gas flow with chemical reactions. Due to high computational costs only simple chemical reaction mechanisms are used here.
\end{abstract}

Keywords: CFD, RDE, rotating detonation engine, AMR, REFLOPS, hydrogen

\section{Introduction}

The detonation is a phenomenon which has been known for over a hundred years. This process of rapid combustion is generally understood, however the details of the shock structure and chemical mechanism of the detonation is still a subject of intense research due to its practical importance. Recently, the study on the RDE has given a new impulse for science of detonation. Because of geometrical complexity of the spinning and rotating detonation, new experimental and numerical tools are necessary. Especially very robust codes for simulating the rotating detonation engine in complex geometries are important. They should allow resolving details of the flow field which are unavailable from analysis of the experimental results. On the other hand, when they are validated, they could be a useful tool for optimisation of the RDE in the future.

This paper describes the development of a computational code REFLOPS USG based on the RANS equations with chemical reactions for semi-ideal multi-component gas to predict the structure and dynamics of three-dimensional unsteady detonation as it occurs in the RDE. The code has been written in FORTRAN 90 programming language. The acronym REFLOPS USG stands for 'REactive FLOw solver for Propulsion Systems on UnStructured Grids'.

\section{Computational model}

The semi-ideal gas model is used in REFLOPS USG. The gas is multi-component with $n_{s p}$ number of components: 


$$
\frac{p}{\rho}=R T, \quad \rho=\sum_{i=1}^{n_{s p}} \rho_{i}, \quad y_{i}=\frac{\rho_{i}}{\rho}, \quad R=\sum_{i=1}^{n_{s p}} \frac{y_{i}}{W_{i}} R_{u},
$$

where specific heat, enthalpy and entropy of the gas components can be described as functions of temperature:

$$
\begin{gathered}
c_{p i}(T)=R \sum_{j=1}^{5} \alpha_{i j} T^{j-1}, \\
h_{i}(T)=R T\left(\sum_{j=1}^{5} \alpha_{i j} T^{j-1}+\frac{\alpha_{i 6}}{T}\right), \\
s_{i}(T)=R\left(\alpha_{i 1} \ln (T)+\sum_{j=2}^{4} \frac{\alpha_{i j}}{j-1} T^{j-1}+\alpha_{i\urcorner}\right),
\end{gathered}
$$

where $\alpha_{i j}$ are coefficients taken from JANAF [1] tables. The temperature is calculated from:

$$
\sum_{i=1}^{n_{s p}} \rho_{i} h_{i}(T)-\rho e=\sum_{i=1}^{n_{s p}} \rho_{i} R_{i}(T) T .
$$

The production rate of chemical compounds $\dot{\omega}_{i}$ is an overall sum of the production and destruction rates for a given chemical compound in all reactions taken into account in the chemistry model:

$$
\dot{\omega}_{i}=\frac{d \rho_{i}}{d t}=W_{i}\left[\sum_{k=1}^{m} \pm v_{i, k} \alpha\left(k_{k, \text { for }} \prod_{j} C_{j}^{v_{j k}}-k_{k, \text { back }} \prod_{j} C_{j}^{v_{j k}}\right)\right],
$$

where:

$W_{i} \quad-i-t h$ specie molar mass,

$v_{i, k} \quad-i$-th specie stoichiometric coefficient in $k-t$ th reaction,

$\alpha=\sum C_{j} \alpha_{j, k}-$ third body coefficient describing collision of three molecules,

$k_{k, f o r}-$ forward reaction rate,

$k_{k, \text { back }}$ - backward reaction rate,

$C_{j} \quad-j-t h$ specie concentration,

$v_{j k} \quad-$ exponent for $j-t h$ specie in $k-t h$ reaction.

The rate of the reactions is described by the Arrhenius equation:

$$
k_{k, f o r}=A_{k} T^{n_{k}} \exp \left(-E_{a, k} / R T\right),
$$

whereas the rate of backward-reactions is taken from the assumption of temporary, local thermodynamic equilibrium:

$$
K_{k}=\frac{k_{k, f o r}}{k_{k, \text { back }}}(R T)^{\Delta v_{k}},
$$

where:

$$
\ln K_{k}=\frac{ \pm v_{i, k} s_{i}^{o}}{R}-\frac{ \pm v_{i, k} \Delta h_{f, i}^{o}}{R T} .
$$

$E_{a, k}$ is an activation energy of the $k$-th reaction, $A_{k}$ pre-exponential constant of the $k$-th reaction, $n_{k}$ the temperature exponent of the $k-t h$ reaction. The model described above is typical in case of modelling gaseous detonations, see [3, 7-9, 14, 18, 19].

Gas dynamics is expressed by $\mathrm{k}-\varepsilon$ standard RANS equations. The modelled conservation equations may be expressed in the following form: 


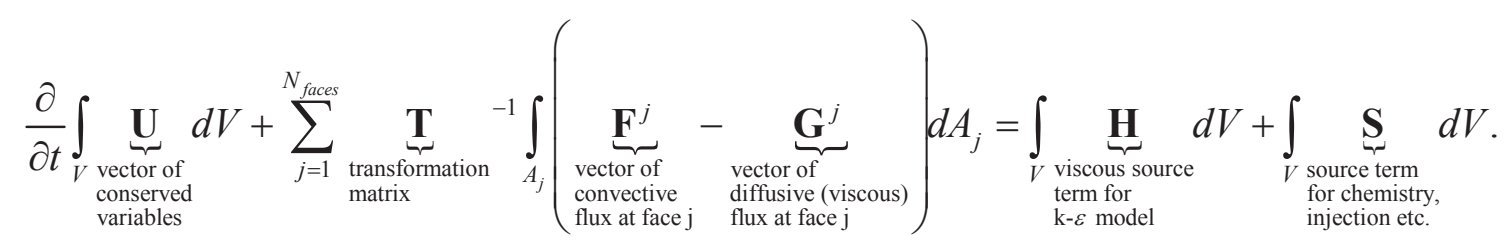

The corresponding vectors are as follows:

$$
\mathbf{U}=\left[\begin{array}{c}
\rho \\
\rho u \\
\rho v \\
\rho w \\
E \\
\rho y_{i} \\
\rho k \\
\rho \mathrm{n}
\end{array}\right], \quad \mathbf{F}^{j}=\left[\begin{array}{c}
\rho \hat{u} \\
\rho \hat{u} \hat{u}+p \\
\rho \hat{v} \hat{u} \\
\rho \hat{w} \hat{u} \\
\hat{u}(E+p) \\
\rho y_{i} \hat{u} \\
\rho k \hat{u} \\
\rho \check{n} \hat{u}
\end{array}\right],
$$$$
\mathbf{G}^{j}=\left[\begin{array}{c}
0 \\
\hat{\tau}_{x x} \\
\hat{\tau}_{x y} \\
\hat{\tau}_{x z} \hat{\tau}_{x x}+\hat{v} \hat{\tau}_{x y}+\hat{w} \hat{\tau}_{x z}-\hat{Q}_{x}-\sum_{i} h_{i} \hat{J}_{i} \\
-\hat{J}_{i} \\
\left(\mu+\frac{\mu_{t}}{\sigma_{k}}\right) \frac{\partial k}{\partial \hat{x}_{j}} \\
\left(\mu+\frac{\mu_{t}}{\sigma_{\check{n}}}\right) \frac{\partial \grave{n}}{\partial \hat{x}_{j}}
\end{array}\right],
$$

$$
\mathbf{H}=\left[\begin{array}{c}
0 \\
0 \\
0 \\
0 \\
0 \\
0 \\
G_{k}-\rho \check{\mathrm{n}}-Y_{m} \\
C_{1 \check{\mathrm{n}}} \frac{\check{\mathrm{n}}_{k}}{k} G_{k}-C_{2 \check{\mathrm{n}}} \rho \frac{\mathrm{n}^{2}}{k}
\end{array}\right], \quad \mathbf{S}=\left[\begin{array}{c}
0 \\
0 \\
0 \\
0 \\
0 \\
\dot{\omega}_{i} \\
0 \\
0
\end{array}\right] .
$$

In the above equations, $G_{k}$ represents the generation of turbulence kinetic energy due to the mean velocity gradients, $Y_{m}$ represents the contribution of the fluctuating dilatation in compressible turbulence to the overall dissipation rate and i.e. $\hat{u}$ is the contravariant velocity component normal to the surface $A_{j}$ defined by $\hat{u}=\overrightarrow{\mathbf{V}} \cdot \overrightarrow{\mathbf{n}}$. The Reynolds stresses $\hat{\tau}_{x x}, \hat{\tau}_{x y}, \hat{\tau}_{x z}$ are provided by equations:

$$
\begin{aligned}
& \hat{\tau}_{x x}=\left(\mu+\mu_{t}\right)\left[\frac{4}{3} \frac{\partial \hat{u}}{\partial \hat{x}_{j}}-\frac{2}{3}\left(\frac{\partial \hat{v}}{\partial \hat{y}_{j}}+\frac{\partial \hat{w}}{\partial \hat{z}_{j}}\right)\right]-\frac{2}{3} \rho k, \\
& \hat{\tau}_{x y}=\left(\mu+\mu_{t}\right)\left(\frac{\partial \hat{u}}{\partial \hat{y}_{j}}+\frac{\partial \hat{v}}{\partial \hat{x}_{j}}\right), \quad \hat{\tau}_{x z}=\left(\mu+\mu_{t}\right)\left(\frac{\partial \hat{u}}{\partial \hat{z}_{j}}+\frac{\partial \hat{w}}{\partial \hat{x}_{j}}\right) .
\end{aligned}
$$

The conservation equations are Favre averaged (density averaged) but the subscripts are omitted here. Note that $\mathbf{F}^{j}=\mathbf{F}^{j}(\mathbf{T} \mathbf{U})$ and $\mathbf{G}^{j}=\mathbf{G}^{j}(\mathbf{T} \mathbf{U})$.

The total energy $E$ is expressed as: 


$$
E=\rho e+\frac{\rho V^{2}}{2}+\rho k
$$

The turbulent viscosity $\mu_{t}$ is modelled by the following equation:

$$
\mu_{t}=\rho C_{\mu} \frac{k^{2}}{\check{n}} \text {. }
$$

The model constants have the following default values:

$$
C_{1 \check{n}}=1.44, \quad C_{2 \check{n}}=1.92, C_{\mu}=0.09, \quad \sigma_{k}=1.0, \quad \sigma_{\check{n}}=1.3 .
$$

Other constants used in the model:

$$
\operatorname{Pr}_{t}=\sigma_{t}=0.85, \quad \mathrm{Sc}_{t}=0.7 \text {. }
$$

The production of turbulent energy is modelled identically for each of k- models. The term representing this production in transport equations is defined as follows:

$$
G_{k}=-\rho \overline{u_{i}^{\prime} u_{j}^{\prime}} \frac{\partial u_{j}}{\partial x_{i}} \stackrel{\substack{\text { Boussinesq } \\ \text { hypothesis }}}{=} \mu_{t} S^{2}
$$

where the modulus of the mean rate-of-strain tensor $S$ is defined as:

$$
S^{2}=2\left(\left[\frac{\partial u}{\partial x}\right]^{2}+\left[\frac{\partial v}{\partial y}\right]^{2}+\left[\frac{\partial w}{\partial z}\right]^{2}\right)+\left(\frac{\partial u}{\partial y}+\frac{\partial v}{\partial x}\right)^{2}+\left(\frac{\partial u}{\partial z}+\frac{\partial w}{\partial x}\right)^{2}+\left(\frac{\partial w}{\partial y}+\frac{\partial v}{\partial z}\right)^{2}
$$

In order to account for effect of the observed decrease in spreading rate with increasing Mach number for compressible mixing and other free shear layers, the dilatation dissipation term $Y_{m}$ is included in the equation for $k$. This term is modelled according to a proposal by Sarkar [11]:

$$
Y_{m}=2 \rho \check{n} M_{t}^{2},
$$

where $M_{t}$ is turbulent Mach number defined as:

$$
M_{t}=\sqrt{\frac{k}{\gamma R T}} .
$$

Viscous heating in the energy equation is defined as:

$$
\hat{Q}_{x}=-c_{p}\left(\frac{\mu}{\operatorname{Pr}_{l}}+\frac{\mu_{t}}{\operatorname{Pr}_{t}}\right) \frac{\partial T}{\partial \hat{x}_{j}}=-\left(\lambda+\frac{\mu_{t} c_{p}}{\operatorname{Pr}_{t}}\right) \frac{\partial T}{\partial \hat{x}_{j}} .
$$

The mass transfer due to diffusion:

$$
\hat{J}_{i}=-\left(\rho D_{i, m}+\frac{\mu_{t}}{S c_{t}}\right) \frac{\partial y_{i}}{\partial \hat{x}_{j}}-\frac{D_{T, i}}{T} \frac{\partial T}{\partial \hat{x}_{j}},
$$

where $D_{i, m}$ is the diffusion coefficient of specie i-th in the mixture and $D_{T, i}$ is thermal diffusion coefficient (Soret) which may be calculated from [6]:

$$
D_{T, i}=-2.59 \times 10^{-7} \cdot T^{0.659} \cdot\left[\frac{M_{w, i}^{0.511} x_{i}}{\sum_{l=1}^{n} M_{w, l}^{0.511} x_{l}}-y_{i}\right] \cdot\left[\frac{\sum_{l=1}^{n} M_{w, l}^{0.511} x_{l}}{\sum_{l=1}^{n} M_{w, l}^{0.489} x_{l}}\right] .
$$

In order to integrate viscous flux $\mathbf{G}^{j}$ and viscous source term $\mathbf{H}$ the time splitting technique is used. The first step is time integration of convective inviscid flux $\mathbf{F}^{j}$ via any explicit method. The inviscid fluxes $\mathbf{F}^{j}$ are given by HLLC Riemann solver $[15 ; 16]$ in conjunction with WAF-TVD second order scheme $[12 ; 13 ; 17]$ or using gradient based second order solution reconstruction [2]. Then the viscous flux and viscous source term can be integrated by use of explicit Euler method:

$$
\mathbf{U}_{i}^{n+1}=\mathbf{U}_{i}^{n}+d t_{d i f f} \frac{1}{\left|V_{i}\right|} \sum_{j=1}^{N_{\text {faces }}} A_{i j} \mathbf{T}^{-1} \mathbf{G}^{j}, \quad \mathbf{U}_{i}^{n+1}=\mathbf{U}_{i}^{n}+d t_{\text {diff }} \mathbf{H} .
$$


In order to deliver the timestep for integration of viscous part of the solution of RANS equations (diffusive part timestep $d t_{\text {diff }}$ ), the following procedure is involved [20]:

$$
d t_{d i f f}=\min \left(d t_{\mu}, d t_{D_{i, m}}, d t_{D_{T, i}}, d t_{\lambda}\right),
$$

where:

$$
\begin{aligned}
& d t_{\mu}=\min _{1}^{N_{\text {faces }}}\left(\frac{\Delta x^{2}}{2} \frac{3 \rho}{4\left(\mu+\mu_{t}\right)}\right), d t_{D_{i, m}}=\min _{1}^{N_{\text {faces }}}\left(\Delta x^{2} \frac{1}{\max _{1}^{N_{\text {species }}}\left(D_{i, m}\right)}\right), \\
& d t_{D_{T, i}}=\min _{1}^{N_{\text {ffaces }}}\left(\Delta x^{2} \frac{1}{\max _{1}^{N_{\text {species }}}\left(D_{T, i}\right)}\right), d t_{\lambda}=\min _{1}^{N_{\text {faces }}}\left(\frac{\Delta x^{2}}{2} \frac{\rho c_{v}}{\lambda}\right),
\end{aligned}
$$

and $\Delta x$ denotes characteristic cell size $\Delta x \approx V / A_{\text {face }}$.

When the computed timestep of diffusion $d t_{d i f f}$ is less than timestep of convection $d t_{\text {conv }}$ the solution is integrated in time marching substeps until the proper time is reached. Otherwise if $d t_{d i f f}$ is higher than $d t_{\text {conv }}$, the solution is advanced by convective timestep.

It is worth to mention that from initial analyses of the RDE combustion chamber, the time scale of convection is much less than the diffusion time scale. Therefore, in practical cases, for highspeed flows, like in the RDE case, the timestep of convection should limit the temporal resolution of the solution. The boundary layer effects are neglected here since the slip walls are assumed, therefore viscous effects only play the role in mixing processes in the combustion chamber (nearby injection zone).

\section{Simulation example}

In the presented simulation the numerical cost was the driving factor and a single-step reversible global reaction mechanism was chosen [4]:

$$
2 \mathrm{H}_{2}+\mathrm{O}_{2}<=>\mathrm{H}_{2} \mathrm{O}+\mathrm{H}_{2} \mathrm{O}, E_{a}=1.2 e 4 \frac{\mathrm{cal}}{\mathrm{mole}}, \quad n=0, \mathrm{~A}=2 \mathrm{e} 17[\text { mole }, \mathrm{cm}, \mathrm{s}, \mathrm{K}] .
$$

This mechanism was checked against the Petersen 21-step mechanism [10] in case of 1D simulation of propagating detonation wave [12].

An attempt for the simulation of the RDE engine was performed for the following data:

- $\quad$ inlet pressure $=3$ bar, Inlet temperature $=320 \mathrm{~K}$,

- $\quad$ outlet pressure $=1$ bar, Outlet temperature $=300 \mathrm{~K}$,

- $\quad$ stoichiometric hydrogen-air mixture (premixed),

- mesh size: min. $0.3 \mathrm{~mm}$ (app.1400000 elements).

The chamber is presented below in Fig. 1. Its total length equals $320 \mathrm{~mm}$ and characteristic heights $\mathrm{h}_{1}, \delta, \mathrm{h}_{2}, \mathrm{~h}_{3}$ are $27,3.8,23,17.5 \mathrm{~mm}$ respectively. The internal diameter equals $150 \mathrm{~mm}$.
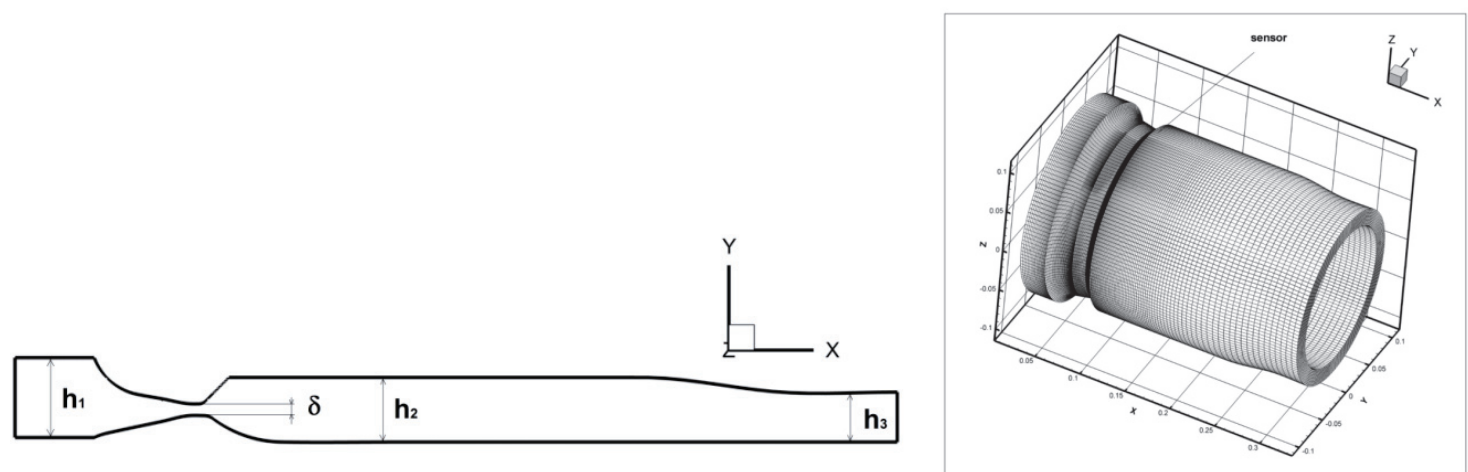

Fig. 1. Left: Sketch of chamber cross section. Flow direction is from left to right. Right: Mesh and sensor location 
In this case one detonation wave was obtained (Fig. 2). The maximum pressure at the detonation wave front equals $15 \mathrm{~atm}$. The wave has a velocity $1617 \mathrm{~m} / \mathrm{s}$, which is close to the Chapman-Jouguet detonation velocity $(1984 \mathrm{~m} / \mathrm{s})$ but lower by $18 \%$. Similar discrepancy has been observed in experiments $[4 ; 5]$. Several explanations could be given to this fact:

- the fresh mixture can partially mix with the detonation products and do not react,

- because of the expansion fan behind the detonation wave the parameters of the gas behind the front are lower comparing to $1 \mathrm{D}$ detonation and thus the wave speed is lower due to wider energy expansion,

- the fresh mixture gain some speed in circumferential direction, so the detonation wave propagates in counter-flowing fresh mixture.

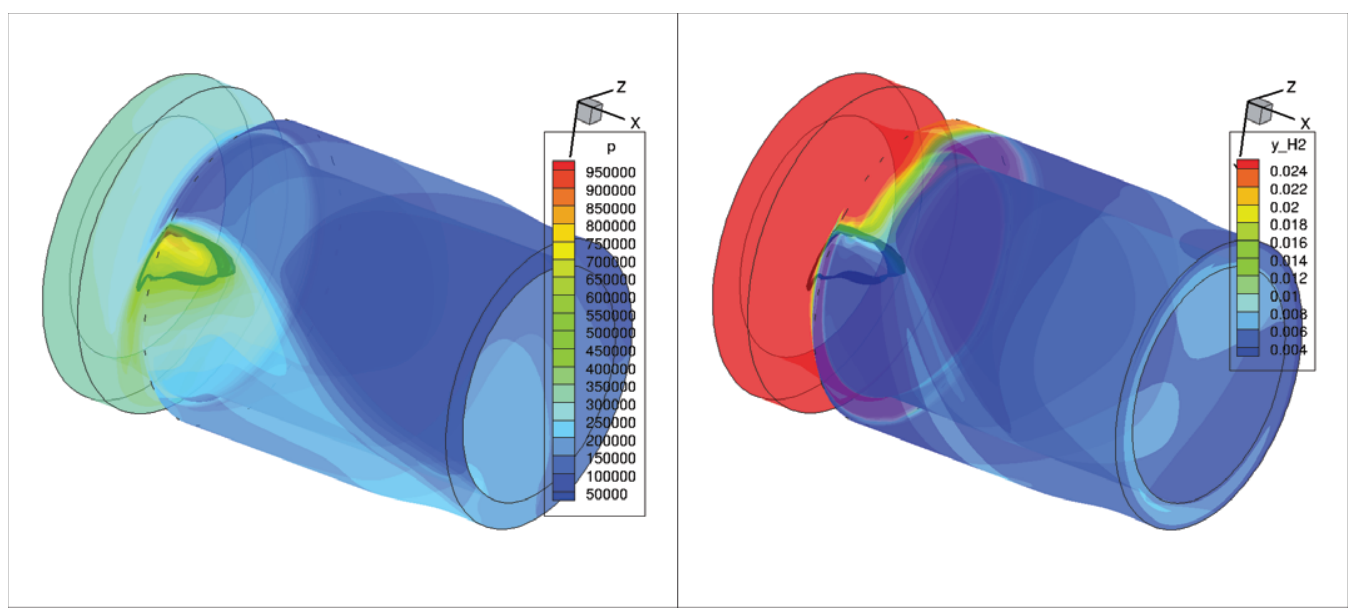

Fig. 2. Detonation wave propagation. Left: Pressure contours and isosurface of the pressure for $p=5$ bar. Right: Contours of mass fractions of $\mathrm{H} 2$ and isosurface of the pressure for $p=5$ bar

The pressure and temperature variation in time is presented in Fig. 3. The numerical sensor was placed nearby to the throat section (see Fig. 1 on right). The sampling frequency was equal to one timestep. The plots indicate that the wave is initially more intense, because the initial pressure in the region of sensor is at the level of 1.5 bar. After short period the pressure drops due to general change of the flow pattern in the nozzle. As a consequence the pressure peaks are also lower. Very important feature of the rotating detonation is its stability. Notwithstanding the pressure peaks slightly vary, the detonation velocity and the period of rotation is very stable. The detonation also generates similar temperature peaks. The peaks correspond to period when the detonation products pass over numerical sensor. The low temperature is the temperature of fresh unburnt mixture.

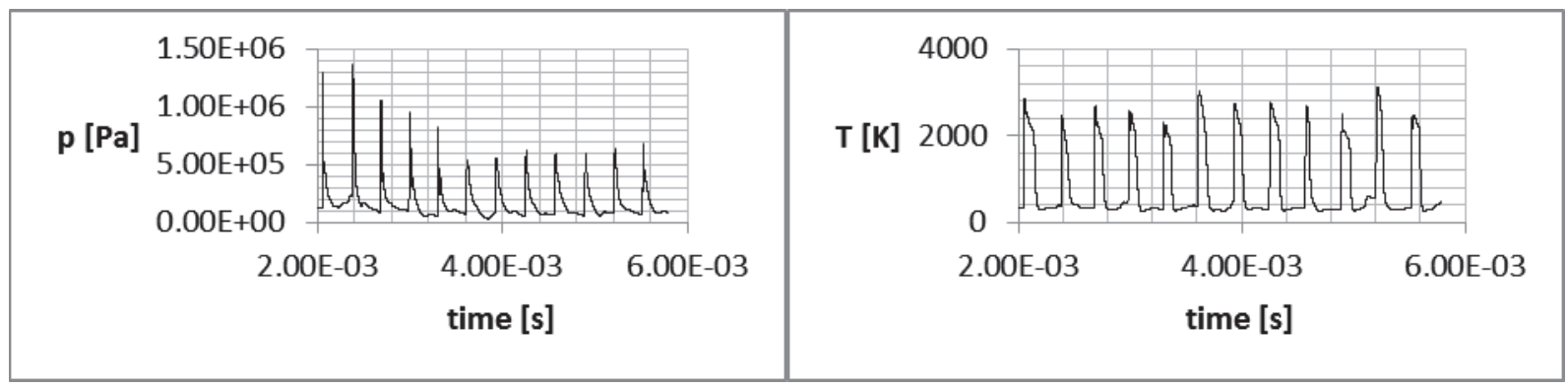

Fig. 3. Left: Pressure reading from numerical sensor. Right: Tempereture reading from numerical sensor

\section{Conclusions}

A short overview of the computational simulation model of the RDE combustion chamber has been presented in this paper. The proposed model has been successfully tested against the real 
geometry and operating conditions of the detonation chamber. From the numerous numerical and experimental analyses we find out that the model is able to accurately predict:

- shock waves dynamics,

- mixing processes of oxidiser and fuel in the RDE combustion chamber,

- flow dynamics in the RDE combustion chamber,

- detonation wave propagation process in the RDE combustion chamber.

More examples of the RDE combustion chamber simulations for various working conditions and different chamber geometries are included in PhD theses of Swiderski [12] and Folusiak [2].

\section{Acknowledgement}

This work was conducted in frame of project UDA-POIG.01.03.01-14-071 'Turbine engine with a detonation combustion chamber' supported by EU and Ministry of Regional Development, Poland.

\section{References}

[1] Chase, M. W., et al., JANAF Thermochemical Tables, $3^{\text {rd }}$ ed. J. Phys. Chem. Ref. Data., Vol. 15. Suppl. 1, 1985.

[2] Folusiak, M., Development of simulation methods of rotating detonation in complex geometries, PhD diss., WUT, Warsaw 2013.

[3] $\mathrm{Hu}, \mathrm{X}$. Y., et al., The structure and evolution of a two-dimensional H2/O2/Ar cellular detonation, Shock Waves, Vol. 14., pp. 37-44, 2005.

[4] Kindracki, J., et al., Experimental and numerical study of the Rotating Detonation Engine in hydrogen-air mixtures, Progress in Propulsion Physics, Vol. 2, pp. 555-582, 2011.

[5] Kindracki, J., Experimental research and numerical simulations of the inititiation of gaseous rotating detonation, PhD diss., WUT, Warsaw 2008.

[6] Kuo, K. K. Y., Principles of Combustion, 2nd edition, John Wiley and Sons, New York 2005.

[7] Leblanc, J. E., Lefebvre, M. H., Fujiwara, T, Detailed Flowfields of a RAMAC Device in H2O2 Full Chemistry, Shock Waves, Vol. 6., pp. 85-92, 1996.

[8] Lefebvre, M. H., Fujiwara, T., Robust Euler Codes for Hypersonic Reactive Flows, Memoirs of the School of Engineering, Vol. 26, pp. 1-54, Nagoya 1994.

[9] Milanowski, K., et al., Numerical Simulation of Rotating Detonation in Cylindrical Channel, 21 st ICDERS, Poitiers 2007.

[10] Petersen, E. L., Hanson, R. K., Reduced Kinetics Mechanisms for Ram Accelerator Combustion, Journal of Propulsion and Power, Vol. 15, pp. 591-600, 1999.

[11] Sarkar, S., Balakrishnan, L., Application of a Reynolds-Stress turbulence model to the compressible shear layer, ICASE Report 90-18, NASA CR 182002, Hampton 1990.

[12] Swiderski, K., Numerical modelling of the rotating detonation combustion chamber, $\mathrm{PhD}$ diss., WUT, Warsaw 2013.

[13] Toro, E. F., A weighted average flux method for hyperbolic conservation laws, Proc Roy Soc Lond A., Vol. 423, pp. 401-418, 1989.

[14] Toro, E. F., Riemann Solvers and Numerical Methods for Fluid Dynamics, $3^{\text {rd }}$ edition, Springer, Berlin 2010.

[15] Toro, E.F., Spruce, M., Speares, W., Restoration of the Contact Surface in the HLL-Riemann Solver, Technical Report CoA-9204, Department of Aerospace Science, Collegue of Aeronautics, Cranfield Institute of Technology, UK 1992.

[16] Toro, E. F., et al., Restoration of the Contact Surface in the HLL-Riemann Solver, Shock Waves, Vol. 4, pp. 25-34, 1994.

[17] Toro, E. F., The weighted average flux method applied to the Euler equations, Philos Trans Roy Soc Lond A., Vol. 341, pp. 499-530, 1992. 
[18] Tsuboi, N., Katoh, S., Hayashi, A. K., Three-dimensional numerical simulation for hydrogen/air detonation: Rectangular and diagonal structures, Proceedings of the Combustion Institute, Vol. 29, pp. 2783-2788., Pittsburg 2002.

[19] Zeldovich, Y. B., Kompaneets, A. S., Theory of Detonation, Academic Press, New York \& London 1960.

[20] Ziegler, J. L., et al., An adaptive high-order hybrid scheme for compressive, viscous flows with detailed chemistry, J. Comput. Phys., Vol. 230, pp. 7598-7630, Pasadena 2011. 